\title{
REVIEW ON MELATONIN IS A NOVEL MOLECULE FOR MANAGEMENT OF VARIOUS DISORDERS Shivakumar S. Ladde ${ }^{1,3}$ Bhusnure O. $\mathbf{G}^{2}$. ${ }^{1}$ Reseach Scholar, Research Center of Channabasweshwar Pharmacy College, Latur, Maharashtra, India ${ }^{2}$ Dept. of Quality Assurance, Channabasweshwar Pharmacy College (Degree), Latur, Maharashtra, India ${ }^{3}$ Dept. of Pharmacology, Shivlingeshwar College of Pharmacy, Almala, Dist-Latur, Maharashtra, India
}

\section{Article Info: Received 14 April 2020; Accepted 13 May 2020}

DOI: https://doi.org/10.32553/jbpr.v9i3.754

Corresponding author: Shivakumar S. Ladde

Conflict of interest statement: No conflict of interest

\begin{abstract}
Melatonin is $\mathrm{N}$-acetyl-5-methoxytryptamine and is an indoleamine. It is synthesized in the pineal gland by the conversion of tryptophan to serotonin, which is acetylated by $\mathrm{N}$-acetyltransferase (NAT) to $\mathrm{N}$-acetyserotonin. $\mathrm{N}$ acetylserotonin is subsequently converted to melatonin by the enzyme hydroxyindole-omethyltransferase. Melatonin receptors are G-protein coupled receptors and mainly classified into $\mathrm{MT}_{1}$ (Mel1a) and $\mathrm{MT}_{2}$ (Mel1b). Melatonin is an ubiquitous molecule, which has been found not only in the human pineal gland but also in vegetables and their fruits and seeds, medicinal herbs, and fermented products such as Piper nigrum, cherry, Kiwifruit, grapes, walnut, etc. In the present research we review the beneficial effect of Melatonin for management of various disorders on basis literature survey available on PubMed, science direct, magazine, e-journal, etc. From the review it conclude that the melatonin has been assessed as a treatment of ocular diseases, blood diseases, gastrointestinal tract diseases, cardiovascular diseases, diabetes, rheumatoid arthritis, fibromyalgia, chronic fatigue syndrome, infectious diseases, neurological diseases, sleep disturbances, aging and depression. Melatonin has been also used as a complementary treatment in anaesthesia, hemodialysis, in vitro fertilization and neonatal care. In the future, analogues of melatonin may be of value in treating various chronic disorders.
\end{abstract}

Keywords: Melatonin, N-acetylserotonin, Piper nigrum, Diabetes, Depression, Hemodialysis

\section{Introduction}

Melatonin is $\mathrm{N}$-acetyl-5-methoxytryptamine and is an indoleamine ${ }^{1,2}$. It is synthesized in the pineal gland by the conversion of tryptophan to serotonin, which is acetylated by $\mathrm{N}$-acetyltransferase (NAT) to $\mathrm{N}$ acetyserotonin. $\mathrm{N}$-acetylserotonin is subsequently converted to melatonin by the enzyme hydroxyindoleomethyltransferase. The pineal hormone production is dependent on the light-dark cycle because of circadian changes in the activity of NAT, the pineal rate limiting enzyme $^{3}$ (Figure 01).

The melatonin effects are mediated by the specific high affinity receptors localized in plasma membranes and coupled to guanosine triphosphate-glutamyl transpeptidase-binding proteins $6^{4}$. Furthermore, investigations of the ovine pars tuberalis demonstrated that melatonin receptors couple both to pertussis-toxinsensitive and cholera-toxin sensitive components, which are involved in the inhibition of CAMP, mediated by the melatonin receptors ${ }^{7}$. Recently, cloning of several Gprotein-coupled melatonin receptors has revealed that three melatonin receptor subtypes exist ${ }^{8,9,10,11}$. It has also been demonstrated that melatonin effects are mediated through specific nuclear receptors (orphan ROR-RZR receptors) and in some cases melatonin can act without receptors, too ${ }^{12,13}$. A significant factor of endogenous melatonin availability is the age. It has been reported that aging is associated with progressive reduction of circadian melatonin synthesis in pineal gland. Equally, the onset of many degenerative and proliferative diseases is associated with aging; what remains unclear is whether the increase of these diseases is related to reduce antioxidative protection potentially provided by melatonin ${ }^{14}$.

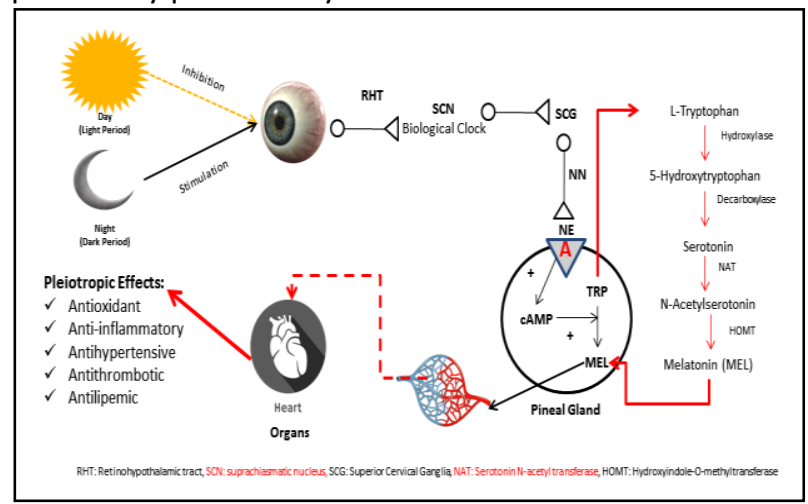

Figure 1: Synthesis of Melatonin in Pineal Gland ${ }^{4,5}$ 
Melatonin influences circadian and seasonal behavior and physiology ${ }^{15}, 16,17$. The nocturnal release of melatonin alters the timing of mammalian circadian rhythms and regulates reproductive changes in response to deviations in day length in seasonally breeding mammals ${ }^{18}$. The efficacy of melatonin has been assessed as a treatment of ocular diseases, blood diseases, gastrointestinal tract diseases, cardiovascular diseases, diabetes, rheumatoid arthritis, fibromyalgia, chronic fatigue syndrome, infectious diseases, neurological diseases, sleep disturbances, aging and depression. Melatonin has been also used as a complementary treatment in anaesthesia, hemodialysis, in vitro fertilization and neonatal care ${ }^{19,20}$

\section{Biosynthesis of melatonin:}

There are four enzymes involved in melatonin synthesis from its amino acid precursor, tryptophan: tryptophan hydroxylase, dopa-decarboxylase, arylalkylamine- $\mathrm{N}$ acetyltransferase, and hydroxyindole-Omethyltransferase. Tryptophan (Trp), taken up from the bloodstream, is the synthetic precursor of all the pineal 5-methoxyindoles. Trp is metabolized into 5-hydroxy-Trp (5-HTP) in the pineal mitochondria by Trp-hydroxylase ${ }^{21}$. The reaction, which occurs in the presence of molecular oxygen, requires (6R)-tetrahydrobiopterin as a cofactor $^{22}$. Tryptophan hydroxylase (TPH) initiates the melatonin biosynthetic pathway, and it is regarded as the rate-limiting enzyme in serotonin $(5-\mathrm{HT})$ synthesis $^{23}$. The second enzyme in the melatonin biosynthetic pathway, dopa decarboxylase, or aromatic L-amino acid decarboxylate (AADC), it is present in large quantities in the cytosolic fraction of the pinealocytes. It is not a limiting factor for decarboxylates the product of TPH (5hydroxytryptophan) to synthesize 5-hydroxytryptamine (5-HT), serotonin ${ }^{24}$.

Arylalkylamine-N-acetyltransferase (AANAT) or 5-HT-Nacetyltransferase is highly localized in the pineal gland and converts serotonin into $\mathrm{N}$-acetylserotonin (NAS), the rate-limiting step in melatonin synthesis. It is this enzyme which controls the circadian rhythm of melatonin production by the pineal gland ${ }^{22}$.

In all vertebrates, enzyme activity is highest at night time and activity decreases very rapidly upon exposure to light. Rhythms in the mammalian pineal gland are set by afferent information derived from the endogenous clock in the hypothalamic suprachiasmatic nucleus $(\mathrm{SCN})^{25}$. Acetylserotonin O-methyltransferase is the last enzyme of the melatonin biosynthesis pathway, catalyzing the transfer of a methyl group from Sadenosyl-L-methionine onto $\mathrm{N}$-acetyl-serotonin, to produce melatonin from $\mathrm{N}$-acetylserotonin (NAS) ${ }^{21,22,26}$.

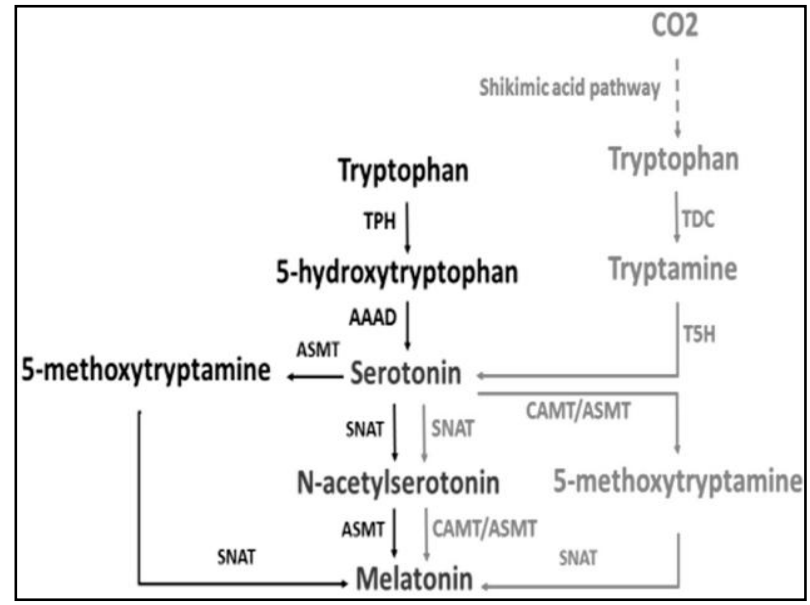

Figure 2: A comparison of the biosynthetic pathways of melatonin in animals and in plants. TPH: tryptophan hydroxylase; AAAD: aromatic amino acid decarboxylase; SNAT: $\quad$ serotonin $\mathrm{N}$-acetyltransferase; $\quad$ ASMT: $\mathrm{N}$ acetylserotonin O-methyltransferase, TDC: tryptophan decarboxylase; T5H: tryptamine 5-hydroxylase, CAMT: caffeic acid O-methyltransferase. Black occurs only in animals; green occurs only in plants; and red occurs in both animals and plants ${ }^{27}$.

\section{Melatonin receptors:}

Melatonin receptors are G-protein coupled receptors and mainly classified into $\mathrm{MT}_{1}\left(\mathrm{Mel}_{1 \mathrm{a}}\right)$ and $\mathrm{MT}_{2}\left(\mathrm{Mel}_{1 \mathrm{~b}}\right)$. Both melatonin receptors have a general structural motif consisting of seven transmembrane (TM)-spanning $\alpha$-helical segments connected by alternating intracellular and extracellular loops, with the amino terminus located on the extracellular side and the carboxyl terminus on the intracellular side. These seven $\alpha$-helical segments contain stretches of 20 to 25 predominantly hydrophobic residues that span the cell membrane ${ }^{28}$.

\section{Melatonin receptor type $1 \mathrm{a}\left(\mathrm{MT}_{1}\right)$ receptors:}

It is encoded in human chromosome \#4 and consists of 351 amino acids. MT1 receptor constitutes adenylate cyclase inhibition by binding to various G-proteins. MT1 receptors are commonly found in human skin. During aging process and Alzheimer's disease, the expression of MT1 receptor in suprachiasmatic nucleus (SCN) and cortex decreases. MT1 receptors reduce the neuronal discharge rate in SCN and suppress prolactin secretion ${ }^{29} . \mathrm{MT}_{1}$ melatonin receptors modulate neuronal firing, arterial vasoconstriction, cell proliferation in cancer cells, and reproductive and metabolic functions ${ }^{30}$. By using recombinant melatonin receptors it has been shown that the $\mathrm{MT}_{1}$ melatonin receptor is coupled to different $\mathrm{G}$ proteins that mediate adenylyl cyclase inhibition and phospholipase $\mathrm{C} 8$ activation $^{31,32}$. $\mathrm{MT}_{1}$ melatonin receptor has the following functions 
a) Inhibition neuronal firing (suprachiasmatic nucleus)

b) Phase shift of onset of circadian rhythm of running wheel activity.

c) Regulation of photoperiodic information.

Endogenous agonists: Melatonin

Agonist: Ramelteon, Agomelatine

Antagonist: Luzindole, PDOT, S29434

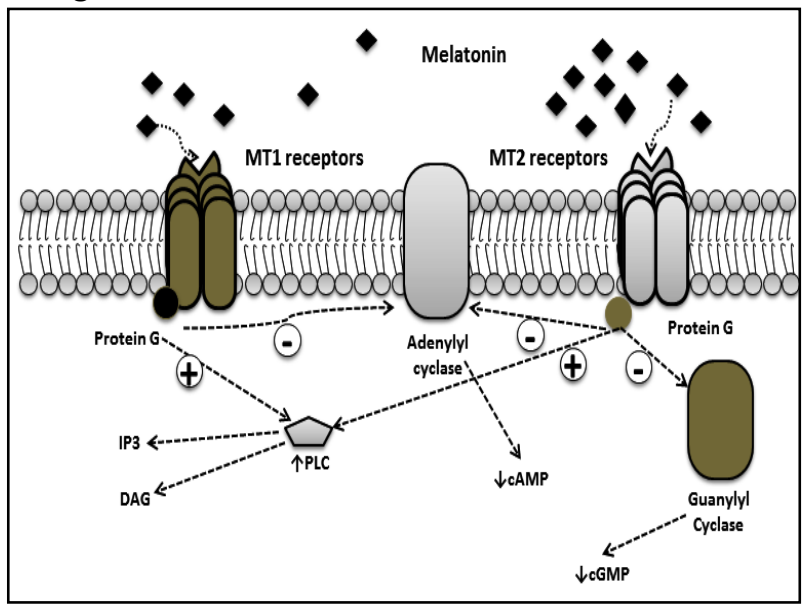

Figure 3: Mechanism of Melatonin on Melatonin receptors $^{33}$

\section{Melatonin receptor type $1 \mathrm{~b}\left(\mathrm{MT}_{2}\right)$ receptor:}

It is encoded in human chromosome \#11 and consists of 363 amino acids. MT2 receptor creates adenylate cyclase inhibition by binding to various G-proteins. Additionally, it inhibits the soluble guanylyl cyclase pathway. Through melatonin receptor activation, adenylate cyclase inhibition occurs and the production of cyclic AMP (cAMP) is reduced. In the skin, MT2 receptors are located within normal and malign melanocytes and eccrine sweat glands. MT2 receptors inhibit GABA-A receptor-related functions in the hippocampus in rats ${ }^{29}$. Activation of $\mathrm{MT}_{2}$ melatonin receptors phase shift circadian rhythms of neuronal firing in the suprachiasmatic nucleus, inhibit dopamine release in retina, induce vasodilation and inhibition of leukocyte rolling in arterial beds, and enhance immune responses ${ }^{30}$. The $\mathrm{MT}_{2}$ receptor is also coupled to inhibition of adenylyl cyclase and additionally it inhibits the soluble guanylyl cyclase pathway ${ }^{31} . \mathrm{MT}_{2}$ melatonin receptor has the following functions

a) Phase shift of the peak of the circadian rhythm of neuronal firing (suprachiasmatic nucleus)

b) Inhibition dopamine release (rabbit retina)

c) Inhibition insuline relase (pancreatic islets) and others.

Endogenous agonists: Melatonin

Agonists: Agomelatine, Ramelteon

Antagonist: Luzindole (Selective), PDOT, S29434

Nutritional source of melatonin:
Melatonin is an ubiquitous molecule, which has been found not only in the human pineal gland but also in vegetables and their fruits and seeds, medicinal herbs, and fermented products such as Grape berries (skin), Spanish wines, Italian wines, Extra virgin olive oil (D. O.), nuts, tomatoes, beetroots, cucumber, banana, strawberry, cherry, apple, Malus, Kiwifruit, Pineapple, Ananas, orange, grapes, walnut, pistachio, bread, cocoa powder, green coffee, mustard seeds, feverfew, St John's Wort, Piper nigrum (Family Piperaceae), B. ramiflora (Family Euphorbiaceae), S. glandiflora (Family Fabaceae), M. charantia (Family Cucerbitaceae), S. tora (Family Fabaceae), S. sesban (Family Fabaceaue)beer, etc., at concentrations varying between $5 \mathrm{pg} / \mathrm{g}$ or $\mathrm{mL}$ to $230 \mu \mathrm{g} / \mathrm{g}$ or $\mathrm{mL}^{34,35,36,37}$

\section{Medicinal approaches for melatonin:}

Melatonin is a hormone secreted from the pineal gland at night. Its peak levels in the dark are associated with age as well as various illnesses. Melatonin plays roles in regulating sleep-wake cycle (figure 01), pubertal development and seasonal adaptation, memory, control of body posture and balance. Melatonin has antinociceptive, antidepressant, anxiolytic, antineophobic (being afraid of new things) and locomotor activity-regulating effects. There are neuroprotective, anti-inflammatory, pain-modulating, blood pressure-reducing, retinal, vascular, seasonal reproductive, ovarian physiology, osteoblast differentiation, anti-tumor and antioxidant effects of melatonin ${ }^{29}$. The goal of present is to review the importance of melatonin in management of various disorders.

\section{Melatonin and Blood Sugar Level:}

Diabetes is a group of metabolic diseases characterized by hyperglycemia resulting from defects in insulin secretion, insulin action, or both. The chronic hyperglycemia of diabetes is associated with long-term damage, dysfunction, and failure of different organs, especially the eyes, kidneys, nerves, heart, and blood vessels $^{38}$. The current classifications for diabetes mellitus type 1-4 are described and the main features of type 1 and type 2 diabetes are compared to allow for better discrimination between these diabetes by correct biochemical diagnosis during fasting and oral glucose tolerance tests as well as the use of hemoglobin $A_{1} C$ $\left(\mathrm{HbA}_{1} \mathrm{c}\right)^{39}$. Melatonin decreases as well the levels of cholesterol, triglyceride, low density lipoprotein, sialic acid, glucose, GSH and might regulate the activities of antioxidant enzymes in diabetic disease.

Melatonin influences insulin secretion both in vivo and in vitro through the $\mathrm{MT}_{1}$-and $\mathrm{MT}_{2}$-receptor-mediated activity. Melatonin through the $\mathrm{IP}_{3}$ pathway is mediated by $\mathrm{Gq}$-proteins, phospholipase $\mathrm{C}$ and $\mathrm{IP}_{3}$, which mobilize 
$\mathrm{Ca}^{2+}$ from intracellular stores, with a resultant increase in insulin. Insulin secretion in vivo, as well as from isolated islets, exhibits a circadian rhythm. This rhythm, which is apparently generated within the islets, is influenced by melatonin, which induces a phase shift in insulin secretion. The observation of the circadian expression of clock genes in the pancreas could possibly be an indication of the generation of circadian rhythms in the pancreatic islets themselves. Melatonin influences diabetes and associated metabolic disturbances. The diabetogens, alloxan and streptozotocin, lead to selective destruction of $\beta$-cells through their accumulation in these cells, where they induce the generation of ROS. $\beta$-cells are very susceptible to oxidative stress because they possess only low antioxidative capacity. Certain results suggest that melatonin in pharmacological doses provides protection against ROS. Finally, melatonin levels in plasma, as well as the arylalkylamine- $\mathrm{N}$-acetyltransferase (AANAT) activity, are lower in diabetic than in non-diabetic rats and humans. In contrast, in the pineal gland, the AANAT mRNA is increased and the insulin receptor mRNA is decreased, which indicates a close interrelationship between insulin and melatonin ${ }^{40,41,42,43}$.

\section{Melatonin and Plasma Cholesterol:}

Some studies have suggested an action of pineal gland on lipid metabolism and, administration of pineal extracts has been shown to lower the serum, hepatic, adrenal and testicular cholesterol levels. In rabbits, pineal extracts could decrease cholesterolemia, biliary cholesterol and serum phospholipids ${ }^{44}$. Cholesterol lowering effect of melatonin has been considered a potent effect as long term melatonin administration could significantly decrease the plasma cholesterol level and prevent fatty liver in genetic hypercholesterolemic rats $^{45}$. Melatonin suspension $(10 \mathrm{mg} / \mathrm{kg})$, were administered orally to the rats fed HCD for 30 days. Melatonin significantly reduced cholesterol absorption in rats fed on HCD and caused significant decreases in total cholesterol, TG, VLDL- and LDL-cholesterol in the plasma and contents of cholesterol and TG in the liver. The level of HDL cholesterol was significantly increased after melatonin. These results suggested that inhibition of cholesterol absorption caused by melatonin could be a mechanism contributing to the positive changes in plasma cholesterol, lipoprotein profile and the lipid contents in the liver ${ }^{49}$.

Furthermore, a melatonin agonist and antagonist stimulate or lower seasonal obesity in the garden dormouse. The role of melatonin on lipid metabolism is also suggested by the observation of delayed post prandial clearance of triacylglycerol indicating possible lipid intolerance in human subjects under simulated nine hour phase-shifts ${ }^{50,51}$. Melatonin could also prevent hyperlipidemia caused by glucocorticoid administration in rats or by cholesterol rich feed ${ }^{45,50}$. Melatonin itself has been shown to inhibit LDL receptor activity and cholesterol synthesis in human mononuclear leucocytes indicated that melatonin also influences lipoprotein lipase activity, a key regulatory enzyme in circulating triacylglycerol in adipose tissue ${ }^{50,51}$.

\section{Melatonin and antioxidant activity:}

Neurodegeneration is frequently associated with damage by free radicals. However, increases in reactive oxygen and nitrogen species, which may ultimately lead to neuronal cell death, do not necessarily reflect its primary cause, but can be a consequence of otherwise induced cellular dysfunction. However, a rise in radical formation should not only be seen as a cause of further damage, but also as a possible consequence of an otherwise initiated malfunction, e.g., by neuronal or cardiac over excitation, calcium overload, ER stress, protein misfolding or reduced expression of relevant proteins which may end up in enhanced mitochondrial electron leakage. The role of mitochondria should not be misinterpreted in terms of earlier concepts, in which the generation of free radicals was directly related to damage of mitochondrial DNA (mtDNA).

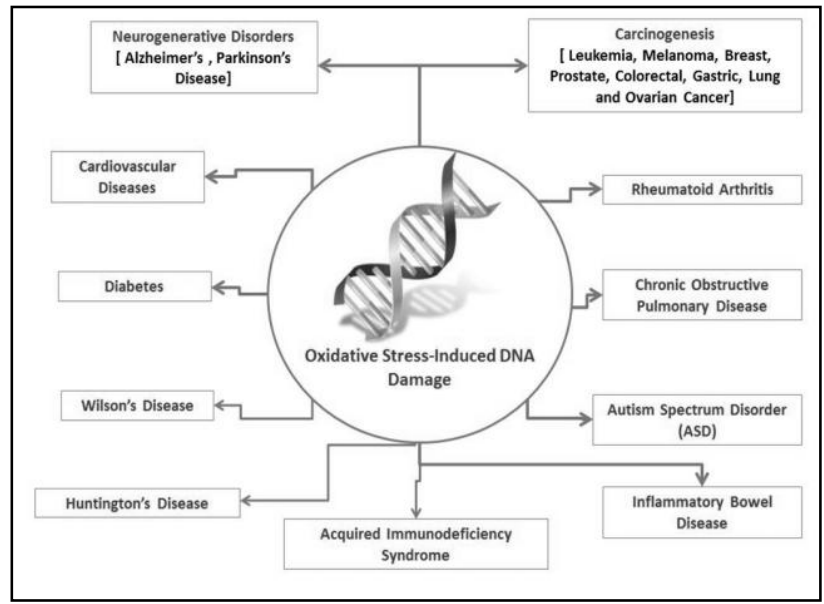

Figure 4: Disorder related to Oxidative Damage to DNA

The enhanced radical generation is that of neuronal over excitation, especially in connection with calcium overload. Glutamate toxicity can be largely explained on this basis, including the secondary rise in the formation of nitric oxide (NO). The vulnerability to calcium imbalance and excitotoxic insults via NMDA receptor activation can be greatly enhanced in a neurodegenerative disorder like Huntington's disease. Insufficiencies in cell respiration, as observed during aging, may also result from a decline in mitochondrial biogenesis $^{52}$.

Melatonin is highly effective in reducing oxidative Damage by enzymatic dismutation of $\mathrm{O2}$, in mitochondria, it involves the stimulation of $\mathrm{SIRT}_{3}$ by 
melatonin; this leads to the deacetylation and activation of SOD. The varieties of melatonin analogues, like cyclic 3-hydroxymelatonin, N1-acetyl-N2-formyl-5methoxykynuramine (AFMK), N1-acetyl-5methoxykynuramine (AMK) and others, which are produced in vivo, also function as antioxidants. The Melatonin also protects mitochondria from elevated mitochondrial $\mathrm{Ca}^{2+}$ stress $^{53,54,55}$. The Melatonin have direct antioxidative protection free radical scavenging activity, and also indirect ways by inhibition of metalinduced DNA damage; protection against non-radical triggers of oxidative DNA damage, continuous protection after being metabolized, activation of antioxidative enzymes, inhibition of pro-oxidative enzymes, and boosting of the DNA repair machinery. The melatonin to exhibit multiple neutralizing actions against diverse threatening factors, together with its low toxicity and its ability to cross biological barriers, are all significant to its efficiency for preventing oxidative damage to $\mathrm{DNA}^{56}$. Melatonin also stimulates antioxidative enzymes like superoxide dismutases (SOD), both MnSOD and CUZnSOD, catalase (CAT), glutathione peroxidase $(\mathrm{GPx})$, glutathione reductase $(\mathrm{GRd})$ and glucose-6-phosphate dehydrogenase (G6PD) ${ }^{57}$. Melatonin has powerful antioxidant, anti-inflammatory and immune properties. These properties function as brain, heart, neurological, cognitive and cancer protection through the reduction of trauma from brain injury; preventing heart muscle damage; neuroprotection; increasing cognitive functioning; and offering cancer support and reducing the toxic effects of chemotherapy ${ }^{58}$.

\section{Melatonin and Cancer:}

Melatonin modulates cancer initiation and progression. The processes by which melatonin may protect against cancer risk, because of its radical scavenging actions and prevents damage to nuclear DNA that results when it encounters reactive oxygen or nitrogen species. It also restrains cancer promotion by limiting the cellular uptake of a growth-promoting fatty acid, i.e., linoleic acid (LA). After its entrance into the cell, LA is converted to 13-hydroxyoctadecadienoic acid (13-HODE) which causes a series of intracellular events that culminate in cancer cell proliferation. Some of these events involve epithelial growth factor (EGF), the phosphorylation and stimulation of downstream signaling molecules, and the mitogen-activated kinases, mitogen-activated protein kinase (MEK) and Extracellular signal-regulated kinase (ERK) 1 and $2^{59}$.

Melatonin exhibits cytotoxic activity in cancer cells. In some cancer cells melatonin lowers their invasive and metastatic status through alterations in adhesion molecules and maintenance of gap junctional intercellular communication. Biochemical and molecular mechanisms of melatonin's oncostatic action may include regulation of estrogen receptor expression and transactivation, calcium/calmodulin activity, protein kinase $\mathrm{C}$ activity, cytoskeletal architecture and function, intracellular redox status, melatonin receptor-mediated signal transduction cascades, and fatty acid transport and metabolism. A major mechanism mediating melatonin's circadian stage-dependent tumor growth inhibitory action is the suppression of epidermal growth factor receptor (EGFR) / mitogen-activated protein kinase (MAPK) activity. This occurs via melatonin receptor-mediated blockade of tumor linoleic acid uptake and its conversion to 13-hydroxyoctadecadienoic acid (13-HODE) which normally activates EGFR / MAPK mitogenic signaling ${ }^{60,61,62,63}$.

Melatonin induces programmed cell death in a wide range of different tumors (breast, gastro-intestinal, hematological, prostate, osteosarcoma, melanoma, kidney, etc...). Melatonin in the nanomolar range activates the intrinsic and/or the extrinsic apoptotic pathway in cancer cells, namely through an increase in the $p 53 / M D M 2 p$ ratio and down regulation of Sirt1 ${ }^{64}$. Melatonin's blockade of BMAL1 expression is associated with the decreased expression of SIRT1, a member of the Silencing Information Regulator family and a histone and protein deacetylase that inhibits the expression of DNA repair enzymes ( 553, BRCA1 \& 2, and Ku70) and the expression of apoptosis-associated genes. In addition to suppressing tumor initiation, melatonin can block or suppress the promotional and progressional phases of cancer development by inhibiting the uptake of linoleic acid and its conversion to 13-HODE, decreasing the mitogenic MAPK/ERK signaling pathway, suppressing/blocking the mitogenic estrogen signaling pathway, repressing ERa expression and transcriptional activity; and potentiating the transcriptional activity of the RARa, RXRa, and VDR to stimulate their growth suppressive and even apoptotic-activating mechanisms ${ }^{65}$.

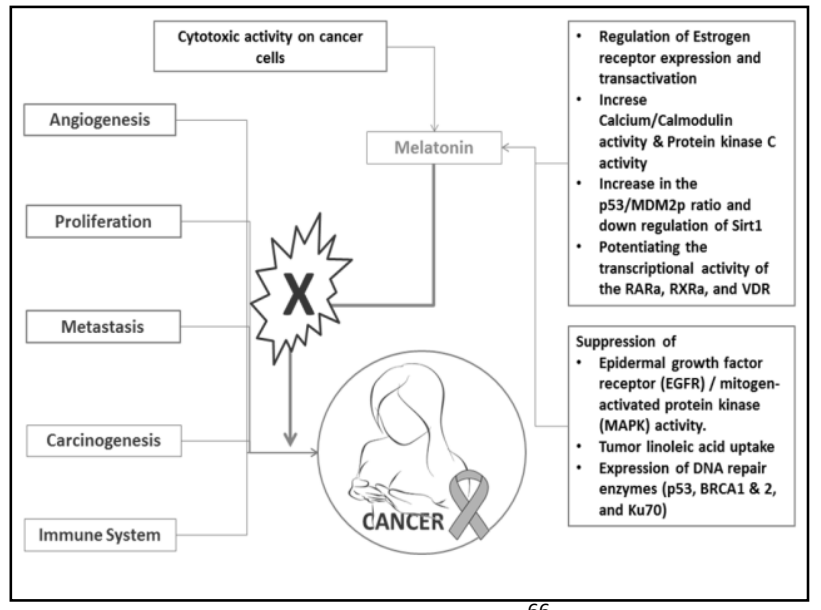

Figure 5: Role of Melatonin in cancer 


\section{Melatonin and cardiovascular disease:}

Nitric oxide (NO) provides cardiovascular protection by reducing blood pressure and via its antiproliferative and antifibrotic actions. Chronic L-NAME ((NLG)-nitro-Larginine methyl ester) administration causes hypertension and target organ damage via the inhibition of NO synthase activity reducing NO production. Moreover, L-NAME-induced deterioration of the vasodilatory function of the renal artery may trigger renin release and renin-angiotensin-aldosterone system (RAAS) activation. Melatonin in the dose of $10 \mathrm{mg} / \mathrm{kg}$ bw can reverse the L-Name induced hypertension by controlling Renin release by a number of neurohumoral stimuli including sympathetic nervous system or oxidative load, both being suppressed ${ }^{67}$. Melatonin confers profound protective effects against ischemiareperfusion injury in various organs, including the heart, liver, and kidney through significantly reduced the level of creatine kinase-MB, by activating silent information regulator 1 (SIRT1) signaling in a receptor-dependent manner, increased expression of $\mathrm{Cu} / \mathrm{Zn}$ superoxide dismutase (SOD-1) and other antioxidant enzymes, basic fibroblast growth factor, insulin-like growth factor 1 , epidermal growth factor, and hepatocyte growth factor, inhibits myocardial apoptosis during myocardial ischemia-reperfusion in rats. It was shown that patients treated with melatonin ( $2-5 \mathrm{mg} /$ day for $7-90$ days) had a decrease in nocturnal SBP as well as DBP. Melatonin reduced the number and area of atheromatous plaques in a rabbit model of atherosclerosis by modulating mitogen-activated protein kinase (MAPK) pathway signal transduction. Melatonin also decreased upstream expression of extracellular signal-related kinase (ERK) and p38. Melatonin has significant effects on ischemiareperfusion injury, myocardial $\mathrm{ClH}$ injury, pulmonary hypertension, hypertension, vascular diseases, valvular heart diseases, and lipid metabolism. As an inexpensive and well tolerated drug, melatonin may be a new therapeutic option for cardiovascular disease ${ }^{68,69,70}$.

In arteriosclerotic patients, the bad cholesterol which blocks arteries is oxidized to produce a more harmful product. Since melatonin is a lipid-loving molecule it can enter the bad cholesterol particles, and stop breakdown of fat molecules to prevent arteries from clogging. A recent study in living organisms showed that melatonin neutralizes free radicals, which are molecule willing to steal electrons from other molecules and cause damage to cells. This reduces the oxidative stress on cells of the heart. Melatonin increased intracellular cGMP level, PKGla expression, p-VASP/VASP ratio and further modulated myocardial Nrf-2-HO-1 and MAPK signaling. However, these effects were blunted by KT5823 (a selective inhibitor of PKG) or PKGl $\alpha$ siRNA except that intracellular cGMP level did not changed significantly. Melatonin ameliorated diabetic $\mathrm{MI} / \mathrm{R}$ injury by modulating Nrf-2-HO-1 and MAPK signaling, thus reducing myocardial apoptosis and oxidative stress and preserving cardiac function. Importantly, melatonin membrane receptors (especially MT2 receptor)dependent cGMP-PKGl $\alpha$ signaling played a critical role in this process ${ }^{71}$. Melatonin protected against $\mathrm{ClH}$-induced myocardial inflammation, fibrosis, and ischemia reperfusion injury by significantly reduced the expression of inflammatory cytokines tumor necrosis factor- $\alpha$ (TNF- $\alpha$ ) and IL-6, IL-12, interferon $¥$ and markers of fibrosis [PC1 and transforming growth factor $\beta$ (TGF $\beta$ )]. Melatonin also increases anti-inflammatory IL-10 $0^{72}$.

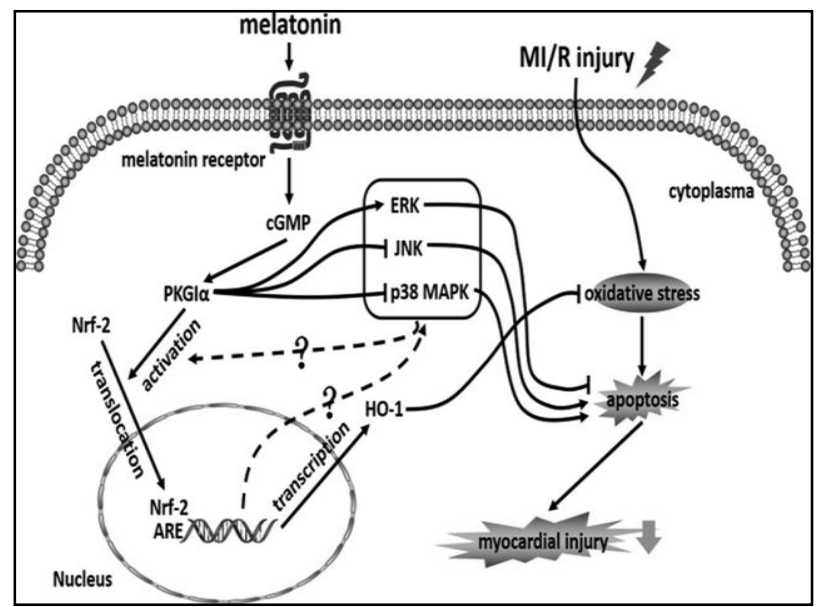

Figure 6: Mechanism of Melatonin in management of Myocardial Injury

\section{Melatonin and Neuropathy Pain:}

The taxane induced neuropathy is thought to be due to aggregation of intracellular microtubules in neuronal cells. It is also thought that taxanes cause intrinsic toxicity and injury to cells. Types of neuropathy caused by taxanes include peripheral neuropathy, motor weakness, myalgias, and arthralgias. About $60 \%-90 \%$ of patients receiving taxanes develop mild-moderate neuropathy and as many as $30 \%$ of treated patients will develop a disabling sensory neuropathy.

In Z. Nahleh et al 2010, study the Melatonin decreases peripheral nerve injury and motoneuron loss on melatonin-treated axotomized rats. Clinical studies have evaluated the role of melatonin for counteracting chemotherapy-toxicity, particularly myelosuppression and immunosuppression. Melatonin has been found to inhibit the production of free radicals, which play a part in mediating the toxicity of chemotherapy ${ }^{73}$.

In a pilot study conducted by Lissoni and colleagues, 80 patients with a variety of metastatic solid tumors received chemotherapy with or without melatonin 20 mg daily. Thrombocytopenia, malaise, asthenia, stomatitis, and neuropathy were less frequent in patients treated with melatonin. The effectiveness of 
chemotherapy was not altered by the addition of melatonin. In another study, 70 patients with non-small cell lung cancer using cisplatin $(20 \mathrm{mg} / \mathrm{m} 2 /$ day intravenously for 3 days) and etoposide $100 \mathrm{mg} / \mathrm{m} 2 /$ day for 3 days received melatonin $20 \mathrm{mg}$ daily or chemotherapy alone ${ }^{74}$.

\section{Melatonin and autism spectrum disorder (ASD):}

ASD refers to a diverse range of neurodevelopmental disorders characterized by social deficits, impaired communication, and stereotyped or repetitive behaviors. Melatonin also plays a crucial role in fetal development. Melatonin can cross physiological barriers, including the blood-placenta barrier, without denaturation, and subsequently influences placental function. During pregnancy, melatonin crosses the placenta and enters the fetal circulation, conveying photoperiodic information to the fetus. Normal melatonin concentrations during pregnancy aid in neuroprotection and normal neurodevelopment of the fetus through the inhibition of excessive oxidative stress in the vulnerable central nervous system. Additionally, as the normal sleep pattern and circadian rhythm are maintained by sufficient melatonin levels, normal melatonin secretion may also influence neurodevelopment. Eventually, the well-known functions of melatonin in neuroprotection and circadian entraining may reduce the risk of $\mathrm{ASD}^{75}$.

\section{Melatonin and Convulsion:}

Melatonin, at 40 and $80 \mathrm{mg} / \mathrm{kg}$, and Agmatine, at 10 and $20 \mathrm{mg} / \mathrm{kg}$, exerted anticonvulsant effects. The anticonvulsant effect of Melatonin (40 and $80 \mathrm{mg} / \mathrm{kg}$ ) was prevented by luzindole $(2.5 \mathrm{mg} / \mathrm{kg})(\mathrm{P}<0.001)$ but not prazosin $(0.5 \mathrm{mg} / \mathrm{kg})$, indicating the possible involvement of ML1/2 receptors in the anticonvulsant effect of melatonin. In conclusion, melatonin and Agmatine exhibit an additive effect in decreasing pentylenetetrazole-induced seizure threshold in mice, probably through ML1/2 receptors ${ }^{76}$.

\section{Melatonin and Cryptorchidism}

Cryptorchidism, a congenital abnormality found in $2 \%-$ $5 \%$ of newborn males, is defined as failure of descent of one (unilateral) or both (bilateral) testes into the scrotum, leaving it in the intra-abdominal position $(2 \%)$, external ring $(9 \%)$, ectopic (11\%) and commonly the inguinal canal $(63 \%)^{77}$. It can be congenital or acquired, and can be caused by environmental and genetic factors ${ }^{78}$.

Cryptorchidism has been associated with spermatotoxicity and oxidative stress while melatonin is a well-known anti-oxidant. This study investigated the possible ameliorative effect of melatonin on cryptorchidism-induced spermatotoxicity and oxidative stress. Cryptorchidism reduced sperm parameters oestradiol, luteinising hormone, follicle stimulating hormone and glutathione peroxidase activity, but increased testosterone and lactate dehydrogenase activity. The cryptorchidism-induced spermatotoxicity and oxidative stress were ameliorated by low dose of melatonin but exacerbated by its high dose ${ }^{79}$.

\section{Conclusion:}

From this review it is concluded that melatonin is potent antioxidant; it may helpful for management of various disorders. The further study is required for molecular mechanism of melatonin involved in the treatment of diabetes, cancer, cardiovascular disorders and autism spectrum disorder and also need epigenetic studies.

\section{References:}

1. Antonio C, Gerardo GM, Carlos A, Dario AC, Russel JR. Melatonin: Potential Functions in the Oral Cavity. J Periodontol 2007; 78(6): 1094-1102.

2. Mulder $\mathrm{H}$, Nagorny $\mathrm{CL}$, Lyssenko $\mathrm{V}$, Groop L. Melatonin receptors in pancreatic islets: good morning to a novel type 2 diabetes gene. Diabetologia 2009; 52 7 7 :1240-1249.

3. Puy H, Deybach JC, Bogdan A, et al. Increased deltaaminolevulinic acid and decreased pineal melatonin production: a common event in acute porphyria studies in the rat. J. Clin. Invest 1996; 97:104-110.

4. Alberto DR, Pedro AG, Pablo A. The role of melatonin in acute myocardial infarction. Frontiers in Bioscience 2012; 17:2433 2441.

5. Rafael B, Sergio M, Javier C, Lourdes F, Cristina S, Rodríguez B, Montserrat R, Carmen B. Assessment of the intake of tryptophan-enriched cereals in the elderly and its influence on the sleep-wake circadian rhythm. Antropologia Portuguesa 2012; 29:113-120

6. Vanececk J. Cellular mechanism of melatonin action. Physiol. Rev. 1998; 78: 687-721.

7. Morgan PJ, Barrett P, Hazlerigg D, Milligan G, Lawson W, Maclean W, Davidson G. Melatonin receptors couple through a cholera toxin sensitive mechanism to inhibit cAMP in the ovine pituitary. J. Neuroendocrinol 1995; 7: 361-369.

8. Reppert SM, Weaver DR, Ebisawa T. Cloning and characterization of a mammalian melatonin receptor that mediates reproductive and circadian responses. Neuron 1994; 13: 1177-1185.

9. Reppert SM, Godson C, Mahle CD, Weaver DR, Slaugenhaupt SA, Gusella JF. Molecular characterization of a second melatonin receptor expressed in human retina and brain: The Mel $1 \mathrm{~b}$ melatonin receptor. Proc. Natl. Acad. Sci. USA 1995; 92: 87348738.

10. Reppert SM, Weaver DR, Godson C. Melatonin receptors step into the light: Cloning and classification of subtypes. Trends Pharmacol. Sci.1996; 17:100-102.

11. Reppert SM. Melatonin receptors, molecular biology of a new family of G protein-coupled receptors. J. Biol. Rhythms 1997; 12: 528-531.

12. Dubocovich, Luzindole ML. (N-0774): a novel melatonin receptor antagonist. J. Pharmacol. Exp. Ther 1988; 246: 902910.

13. Becker Andre, M, Wiesenberg, Schaeren-Wiemers, N, Adre E, Missibach M, Saurat JH, Carlber C. Pineal gland hormone melatonin binds and activates an orphan of the nuclear receptor superfamily. J. Biol. Chem 1994; 269: 28531-28534.

14. Reiter R.J. The aging pineal gland and its physiological consequences. Bioessays 1992; 14: 169-175. 
15. Reiter RJ. Pineal melatonin: cell biology of its synthesis and its physiological interactions. Endocr. Rev 1991; 12: 151-180.

16. Reiter RJ. Interactions of the pineal hormone melatonin with oxygen-centered free radical: A brief review. Braz. J. Med. Biol, Res 1993; 26(11): 11141-1155.

17. Pang SF Lee PPN, Chan YS, Ayre EA. Melatonin secretion and its rhythms in biological fluids. In: Melatonin: Biosynthesis, Physiological Effects, and Clinical Applications, H.H. Yu, R.J. Reiter, eds. CRC Press, Boca Raton 1992; 129-153.

18. Badness TJ, Powers B, Hastings MH, Bittman EL, Goldman BD. The timed infusion paradigm for melatonin delivery: What has it taught as about the melatonin signal, its reception, and the photoperiodic control of seasonal responses? J. Pineal Res 1993; 15: 161-190.

19. Sanchez-Barcelo EJ, Mediavilla MD, Tan DX, Reiter RJ. Clinical Uses of Melatonin: Evaluation of Human Trials. Current Medicinal Chemistry 2010; 17 (19): 2070-95.

20. Sanchez-Barcelo EJ, Mediavilla MD, Reiter RJ. Clinical Uses of Melatonin in Pediatrics. International Journal of Pediatrics 2011; 2011: 1-11.

21. Valerie S, Christophe R. Generation of the Melatonin Endocrine Message in Mammals: A Review of the Complex Regulation of Melatonin Synthesis by Norepinephrine, Peptides, and Other Pineal Transmitters. Pharmacological Review 2003; 55(2):325395.

22. Sumi-Ichinose $C$, Ichinose $H$, Takahashi $E$, Hori $T$, Nagatsu $T$. Molecular cloning of genomic DNA and chromosomal assignment of the gene for human aromatic L-amino acid decarboxylase, the enzyme for catecholamine and serotonin biosynthesis. Biochemistry 1992; 31(8):2229-38.

23. Dax VD, George E. Bentley Seasonal Reproduction in Vertebrates: Melatonin Synthesis, Binding, and Functionality Using Tinbergen's Four Questions. Molecules 2018; 23(652); 1 51.

24. King TS, Steinlechner S. Pineal indolalkylamine synthesis and metabolism: kinetic considerations. Pineal Res Rev 1985; 3:69113.

25. Klein DC, Coon SL, Roseboom PH, Weller JL, Bernard M, Gastel JA, Zatz M, luvone PM, Rodriguez IR, Bégay V, Falcón J, Cahill GM, Cassone VM, Baler R. The melatonin rhythm-generating enzyme: molecular regulation of serotonin $\mathrm{N}$-acetyltransferase in the pineal gland. Recent Prog Horm Res. 1997; 52: 307-57.

26. Kyoungwhan Back, Dun-Xian Tan, Russel J. Reiter. Melatonin biosynthesis in plants: multiple pathways catalyze tryptophan to melatonin in the cytoplasm or chloroplasts. Journal of Pineal Research 2016; 61: 426-437.

27. Dun-Xian Tan, Lucien CM, Eduardo Esteban-Zubero, Zhou and Reiter RJ. Melatonin as a Potent and Inducible Endogenous Antioxidant: Synthesis and Metabolism. Molecules 2015; 20(10): 18886-18906.

28. Margarita LD, Philippe D, Diana NK, David S, Daniel PC, James O. International Union of Basic and Clinical Pharmacology. LXXV. Nomenclature, Classification, and Pharmacology of G ProteinCoupled Melatonin Receptors. Pharmacological Reviews 2010; 62: 343-380.

29. Mucahit E, Halil O, Lutfu O, Muhammed Y, Zekai H, Ahmet Ha. A Review of Melatonin, Its Receptors and Drugs. Eurasian J Med. 2016 Jun; 48(2): 135-141.

30. Margarita LD, Magdalena M. Functional MT1 and MT2 melatonin receptors in mammals. Endocrine 2005; 27(2): 101-110.

31. Charlotte VG, Jorg HS, David RW. Mammalian melatonin receptors: molecular biology and signal transduction. Cell and Tissue Research 2002; 309(1): 151-162.

32. Dubocovich ML. Melatonin receptors: are there multiple subtypes?.Trends Pharmacol. Sci., 1995; 16: 50-56.

33. Anna T, Natascia C, Giampaolo M, Mariusz RW, Gina A, Giampaolo $\mathrm{G}$ and Paolo P. Melatonin as a master regulator of cell death and inflammation: molecular mechanisms and clinical implications for newborn care. Cell Death and Disease 2019; 10(317):1-12.

34. Marcello I, Elena MV, Sara V. Melatonin in traditional Mediterranean diets. Pineal Res. 2010; 49:101-105

35. Xiaoyuan F, Meng W, Yanyun Z, Ping H, Ying D. Melatonin from different fruit sources, functional roles, and analytical methods. Trends in Food Science \& Technology 2014; 37: 21-31.

36. Xiao M, Ya L, Sha L, Yue Z, Ren-You G, Dong-Ping X, Hua-Bin L. Dietary Sources and Bioactivities of Melatonin. Nutrients 2017; 9(367): 1-65

37. Marino BA. Phytomelatonin: Discovery, Content, and Role in Plants Advances in Botany 2014; 2014: 1-11

38. American Diabetes Association. Diagnosis and Classification of Diabetes Mellitus. Diabetes Care 2010; 33(1): s62-s69.

39. Michael R. Diabetes mellitus - Definition, Klassifikation und Diagnose. Wiener klinische Wochenschrift 2016; 128(2): 3740.

40. Elmar P. Melatonin, endocrine pancreas and diabetes. J. Pineal Res. 2008; 44:26-40

41. Patel MM, Ramachandran AV. Seasonal alterations in the weights of pancreas, spleen and uropygeal gland in normal and pinealectomised domestic pigeons, Columba livia. PAVO, 1983; 22: 87-92.

42. Patel $C D$, Asnani MV, Ramachandran AV, Shah RV. Seasonal variation of certain blood parameters in normal and pinealectomised Feral Blue rock pigeon Columba livia. J. Anim. Morphol. Physiol., 1982; 30: 208-216.

43. Patel MM, Ramachandran AV. In vitro influence of hormones on transport of glucose and glycogen in liver and muscle of pinealectomized pigeons, Columba livia. Gmelin. Ind. J. Expt. Biol., 1992; 30: 211-213.

44. Esquifino A, Agrasal C, Velazquez E, Villanua MA, Cardinal DP. Effect of melatonin on serum cholesterol and phospholipid levels, and on prolactin, thyroid-stimulating hormone and thyroid hormone levels, in hyperprolectinemic rats. Life Sci., 1997; 61: 1051-1058.

45. Aoyama, H., Mori, N., Mori, W. Effects of melatonin on genetic hypercholesterolemia in rats. Atherosclerosis., 1988; 69: 269.

46. Saad A, Rehman H. Effect of melatonin on cholesterol absorption in rats. Journal of Pineal Research 2007; 42(3):267271.

47. Le GS, Delagrange P. Atgie C, et al. Effects of both a melatonin agonist and antagonist on seasonal changes in body mass and energy intake in the garden dormouse. Int. J. Obes. Relat. Metab. Disord. 1996; 20: 661-667.

48. Hampton SM, Morgan LM, Lawrence $N$, Anastasiadou T, Norris F, Deacon S, Riberio D, Arendt J. Postprandial hormone and metabolic responses in simulated shiftwork. J. Endocrinol., 1996; 151:259-267.

49. Mori $\mathrm{N}$, Aoyama $\mathrm{H}$, Murase $\mathrm{T}$, Mori W. Antihypercholesterolemic effect of melatonin in rats. Acta Pathol. Jpn. 1989; 39:613-618.

50. Muller-Wieland D, Behnke B, Koopman K, Krone W. Melatonin inhibits LPL receptor activity and cholesterol synthesis in freshly isolated human mononuclear leukocytes. Biochemistry and Biophysics Research Communications. 1994; 203: 416-421.

51. Chapman $\mathrm{C}$. The effects of diet and gastrointestinal hormones on lipoprotein lipase activity and gene expression. PhD. Thesis, University of Surrey, Guldford, UK. 1997.

52. Rudiger H. Neuroprotection by Radical Avoidance: Search for Suitable Agents. Molecules 2009, 14, 5054-5102

53. Russel JR, Dun XT, Sergio RC, Annia G, Xin JZ, Bing X. Mitochondria: Central Organelles for Melatonin's Antioxidant and Anti-Aging Actions. Molecules 2018, 23, 509: 1-25

54. Peng $\mathrm{TI}, \mathrm{Hsiao} \mathrm{CW}$, Reiter RJ, Tanaka M, La YK, Jou MJ. mtDNA T8993G Mutation-induced Mitochondrial Complex V Inhibition Augments Cardiolipin-dependent Alterations in Mitochondrial Dynamics during Oxidative, Ca2+, and Lipid Insults in NARP 
Cybrids: A Potential Therapeutic Target for Melatonin. J. Pineal Res. 2012; 52: 93-106.

55. Reiter RJ, Paredes SD, Korkmaz A, Jou MJ, Tan DX. Melatonin Combats Molecular Terrorism at the Mitochondrial Level. Interdiscip. Toxicol. 2008;1: 127-149.

56. Annia Galano, Dun-Xian Tan and Russel J. Reiter. Melatonin: A Versatile Protector against Oxidative DNA Damage. Molecules 2018; 23(530): 1-36.

57. Russel JR, Dun-xian T, Juan CM, Rosa MS, Josefa L, Zbigniew C. Melatonin as an antioxidant: biochemical mechanisms and pathophysiological implications in humans. Acta Biochemical Polonica 2003; 50 (4): 1129-1146.

58. https://biomedicine.com/articles/2015/02/03/melatoninextraordinary-antioxidant-benefits-beyond-sleep. Date: 23.05.2018

59. Russel JR, Sergio ARC, Dun-Xian T, Dario AC, Lilan $Q$, Shun-Fa $Y$, Kexin X. Review Melatonin, a Full Service Anti-Cancer Agent: Inhibition of Initiation, Progression and Metastasis Int. J. Mol. Sci. 2017; 18(843); 1-47

60. Blask DE, Sauer LA, Dauchy RT. Melatonin as a Chronobiotic / Anticancer Agent: Cellular, Biochemical, and Molecular Mechanisms of Action and their Implications for CircadianBased Cancer Therapy. Current Topics in Medicinal Chemistry 2002; 2(2): 113-132.

61. Giuseppe DB, Fabrizio M, Luciano G, and Luigi DB. Melatonin Anticancer Effects: Review. Int. J. Mol. Sci. 2013; 14(2): 24102430.

62. Brittney J, Nihal A. Melatonin in Cancer Management: Progress and Promise. Cancer Res 2006; 66 (20): 9789-93.

63. Antonio C, Antonio LV, Salvador AS, Joaquin DV, Rafael GDD. Role of Melatonin in Cancer Treatment. Anticancer Research 2012; 32: 2747-2754.

64. Mariano B, Sara P, Alessandra C, Russel JR. Molecular mechanisms of the proapoptotic actions of melatonin in cancer: a review. Expert Opinion on Therapeutic Targets 2013; 17(12): 1483-1496.

65. Steven MH, Tripp F, Shulin X, Lin Y, Tamika D, Lulu M. Molecular Mechanisms of Melatonin Anticancer Effects. Integrative Cancer Therapies 2009; 8(4): 337-346.

66. Mohammad HA, Milad M, Emad G, Marjan F, Mohammad A. Melatonin as a multifunctional anti-cancer molecule: Implications in gastric cancer. Life Sciences 2017; 185:38-45.
67. Fedor S, Tomas B, Kristina K, Kristina R, Silvia A, Stefan Z, Marko $P$, Michaela A, Russel JR, Ludovit P. Effect of Melatonin on the Renin-Angiotensin-Aldosterone System in L-NAME-Induced Hypertension. Molecules 2018; 23: 265:1-15.

68. Hang $S$, Aaron MG, Shen Q. Effects of melatonin on cardiovascular diseases: progress in the past year. Curr Opin Lipidol. 2016; 27(4): 408-413.

69. Lionel H. Opie Sandrine Lecour. Melatonin has multiorgan effects. European Heart Journal - Cardiovascular Pharmacotherapy 2016; 2(4): 258-265.

70. Russel JR, Dun-Xian T, Sergio DP, Lorena FB. Beneficial effects of melatonin in cardiovascular disease. Annals of Medicine 2010; $42(4): 1-20$

71. Li-ming $Y$, Wen-cheng $D$, Xue $D$, Zhi L, Yong $Z$, Xiao-dong $X$, Yin-li $X$, Jian $Z$, Xiong $X$, Jin-song $H, Y u L$, Yang $Y$, Hui-shan W. Melatonin protects diabetic heart against ischemia-reperfusion injury, role of membrane receptor-dependent cGMP-PKG activation. Biochimica Biophysica Acta (BBA) - Molecular Basis of Disease 2018; 1864: 563-578.

72. Necdet FY, etal. Effects of Sleep Quality on Melatonin Levels and Inflammatory Response after Major Abdominal Surgery in an Intensive Care Unit. Molecules 2017; 22(1537): 1-7.

73. Nahleh Z, Pruemer J, Lafollette J, Sweany S. Melatonin, a promising Role in Taxane-Related neuropathy. Clinical Medicine Insights: Oncology 2010; 4: 1-20.

74. Lissoni P, Tancini G, Barni S, et al. Treatment of cancer chemotherapy induced toxicity with the pineal hormone melatonin. Support Care Cancer. 1997; 5: 126-9.

75. Yunho J, Jeonghyun $C$, Jinyoung $W$, Yonggeun $H$. The Relationship between Autism Spectrum Disorder and Melatonin during Fetal Development. Molecules 2018; 23(198); 1-9.

76. Leila M, Hamed S, Abolfazl H, Ahmad RD. The interaction of melatonin and agmatine on pentylenetetrazole-induced seizure threshold in mice. Epilepsy \& Behavior 2011; 22(2): 200-206.

77. Dohle GR, Colpi GM, Hargreave TB, et al. EAU guidelines on male infertility. Eur Urol, 2005; 48: 703-711.

78. Urh K, Kunej T. Molecular mechanisms of cryptorchidism development: update of the database, disease comorbidity, and initiative for standardization of reporting in scientific literature. Andrology 2016; 4: 894-902.

79. Luqman AO, Isiaka AA, Hawau O, Felix O. Low dose of melatonin ameliorates cryptorchidism-induced spermatotoxicity in rats. Journal of the Anatomical Society of India 2017; 66(1): 67-71. 